\title{
Osteomielitis aguda por Cedecea lapagei
}

\section{Acute osteomyelitis by Cedecea lapagei}

\author{
John Lidemberto Cárdenas • Medellín (Colombia)
}

\section{Resumen}

Presentamos un caso de osteomielitis aguda en un paciente con fractura cerrada de cúbito y radio por accidente de tránsito, a quien se le aisló Cedecea lapagei en cultivo de tejido óseo obtenido en cirugía cuando fue llevado para lavado y curetaje. La evolución clínica del paciente y el tratamiento son presentados y discutidos en este reporte. Se realiza una revisión acerca de esta bacteria y los principales tipos de infección que causa y los factores relacionados con su presentación. (Acta Med Colomb 2015; 40: 246-248).

Palabras clave: Cedecea, fractura cerrada, osteomielitis.

\begin{abstract}
A case of acute osteomyelitis in a patient with closed fracture of the ulna and radio by a traffic accident is presented. Cedecea lapagei was isolated in bone tissue culture obtained in surgery when he was brought to washing and curettage. The clinical evolution and treatment are presented and discussed in this report. A review on this bacterium and the main types of infection that it causes as well as factors related to its presentation, are made. (Acta Med Colomb 2015; 40: 246-248).

Keywords: Cedecea, closed fracture, osteomyelitis
\end{abstract}

Dr. John Lidemberto Cárdenas: Internista, Hospital Pablo Tobón Uribe. Medellín (Colombia). Correspondencia. Dr. John Lidemberto Cárdenas. Medellín (Colombia).

E-mail: jolica@hotmail.com Recibido: 22/VIII/2014 Aceptado: 31/VIII/2015

\section{Introducción}

El género Cedecea es un grupo de bacilos Gram negativos anaerobios facultativos, publicado desde 1981 por Grimont y col. (1-4), fermentadores de glucosa, (4), pertenecientes a la familia enterobacteriacea inicialmente clasificados por la CDC como entéricos del grupo 15 (1-4) porque no se adecuaba a ningún otro grupo de esta familia. Su nombre proviene, Cedecea de las siglas CDC ("Center for Diseases Control and Prevention") donde fue descrito inicialmente, y lapagei de "Lapage" en homenaje a Stephen P. Lapage bacteriólogo británico (2).

Infecciones causadas por Cedecea lapagei en general son muy escasas, existen muy pocos reportes en la literatura acerca del papel de este germen en las infecciones, tanto adquiridas en la comunidad como intrahospitalarias, con una mayor importancia en estas últimas. No existen reportes en la literatura de osteomielitis asociadas con este tipo de germen.

El escaso conocimiento de este tipo de germen, su comportamiento epidemiológico y de las infecciones con que se relaciona fueron las principales motivaciones para la realización del reporte de este caso.

\section{Caso}

Paciente de 47 años, conductor de camión, previamente sano, sin antecedentes personales ni familiares importantes.
Sufre trauma por accidente de tránsito, presentando fractura cerrada de cúbito y radio y múltiples excoriaciones, sin otras lesiones.

Consulta inmediatamente luego del accidente por el servicio de urgencias, allí se hace el diagnóstico y es inmovilizado con férula de yeso braquiopalmar y dado de alta. Es reevaluado 48 horas después y se realiza cirugía ambulatoria con reducción abierta con material de osteosíntesis y se deja con vendajes.

Ocho días más tarde consulta por dolor local. Al examen físico se observa edema, calor y eritema asociado a mal olor, el resto del examen físico es normal y no tiene signos de respuesta inflamatoria sistémica.

Se hospitaliza con sospecha de osteomielitis. Se lleva a cirugía para lavado y curetaje óseo bajo anestesia general, el cual se realiza en las primeras 48 horas de su ingreso. Se envía muestra para cultivo de tejido óseo y se inicia empíricamente oxacilina y rifampicina para cubrimiento de S. aureus incluyendo cepa resistente a oxacilina. Es llevado a nuevo lavado a las 96 horas, de acuerdo con los hallazgos operatorios, continúa con curaciones.

El reporte del cultivo de hueso, mediante identificación por Vitek, reportó Cedecea lapagei. Confirmación con método de sensibilidad de difusión en disco, mostró sensibilidad a ertapenem. Se cambia el tratamiento antibiótico 
de acuerdo con el reporte de sensibilidad y es llevado a nuevo lavado por secreción purulenta por herida quirúrgica con reporte de cultivo negativo. En el último lavado no hay signos de infección por lo que se deja con curaciones, completando tratamiento antibiótico 42 días con buena evolución clínica.

\section{Discusión}

Infecciones por cepas del género Cedecea son muy poco frecuentes en la literatura, este género comprende cinco especies, Cedecea lapagei, Cedecea davisae, Cedecea netteri, y dos no nominadas Cedecea sp3 y Cedecea sp5, cada una definida por propiedades fenotípicas e hibridización de DNA (1-4).

Los cultivos de Cedecea son lipasa (+), oxidasa (-) y catalasa $(+)$, es resistente a colistina y cefalotina, propiedades compartidas con Serratia (1-4).

La mayoría de las infecciones por gérmenes del género Cedecea descritas en la literatura son reportes de casos y casi todas por especies diferentes a Cedecea lapagei, principalmente por Cedecea netteri y Cedecea davisae.

Las infecciones por Cedecea lapagei han sido reportadas tanto adquiridas en la comunidad como intrahospitalarias, este caso sugiere una adquisición intrahospitalaria, teniendo en cuenta que se trata de un paciente joven, previamente sano, que recibe una intervención quirúrgica por una fractura cerrada y posterior a ello inicia con un proceso inflamatorio local en el sitio de la intervención. Apoya su papel patógeno, el aislamiento de un sitio estéril y con asepsia quirúrgica adecuada.

Entre las principales infecciones asociadas con la presencia de Cedecea lapagei, están: infecciones de tracto respiratorio, infecciones de sitio operatorio, infecciones urinarias, incluso bacteremia y sepsis secundaria.

El mayor estudio encontrado en la literatura es un estudio en un Hospital público de Brasil, encontraron 52 aislamientos en áreas de hospitalización y cirugía, de ellos 27 fueron por Cedecea lapagei (52\%), con un perfil de sensibilidad menor del $50 \%$ para la mayoría de los antibióticos y para imipenem 95\%. La mayoría de los aislamientos fueron obtenidos en áreas de clínica médica $(25 \%)$, clínica quirúrgica $(20 \%)$, unidad de terapia intensiva $(15 \%)$ y neonatos $(12 \%)$. Los sitios más comunes de aislamiento fueron orina $(50 \%)$, sangre $(20 \%)$, catéter venoso $(15 \%)$ y herida quirúrgica (11\%) (1).

Los reportes de casos han sido en sitios como pulmón, en un paciente con enfermedad pulmonar cavitaria por tuberculosis (3), en infección de herida quirúrgica y bacteremia secundaria en un paciente diabético (4), una peritonitis en un paciente con trasplante de hígado, un caso ventriculitis en un neonato (5), e infección urinaria en un paciente de la unidad de cuidados intensivos (2). Todos los pacientes tenían un estado predisponente que pueden favorecer la aparición de infecciones por gérmenes oportunistas y patógenos emergentes, como Cedecea lapagei en este caso. Este paciente no presentaba ningún factor de riesgo que se asociara con algún estado de inmunosupresión ni enfermedades previas o alteraciones estructurales secundarias. No existen en la literatura reportes de infecciones óseas por Cedecea lapagei en pacientes sin factores de riesgo.

La mortalidad asociada con infecciones por Cedecea lapagei en la literatura es $0 \%$, con evolución clínica y respuesta terapéutica excelente con el tratamiento antibiótico.

Cedecea lapagei sigue siendo un germen muy poco reconocido, tanto en el ambiente clínico como en el laboratorio, y puede confundirse con otras enterobacterias. Los aislamientos reportados se encuentran principalmente en infecciones en el ambiente intrahospitalario.

Preocupa el papel creciente de este tipo de germen como causante de infecciones intrahospitalarias. El tratamiento de elección para este tipo de bacterias no se conoce porque no se ha estudiado el desenlace clínico para diferentes antibióticos a los que la bacteria muestre sensibilidad, debido a que es un aislamiento poco común, pero se muestra en la literatura un perfil de resistencia que ha venido cambiando, encontrándose cepas multisensibles en aislamientos iniciales cuando se comenzó a detectar esta bacteria. Hoy en día se considera una bacteria multirresistente y los estudios más grandes reportan mayor sensibilidad a carbapenémicos, con reportes de cepas de Cedecea productoras de ampC (6). Así mismo, es muy importante la correlación clínica ante el aislamiento de Cedecea, ya que en algunos casos no ha sido claro su papel etiológico y es clasificada dentro de los nuevos géneros de enterobacterias oportunistas, junto a cepas como Tatumella, Rahnella, Kluyvera y Ewingella, y es fundamental definir un manejo antibiótico adecuado por su alto perfil de resistencia a múltiples antibióticos. (1)

Los sistemas automatizados han mostrado mayor sensibilidad en su detección, pero en algunos casos no tienen paneles de antibiograma específicos para determinar adecuadamente el perfil de resistencia, esto dado su escasa identificación, pocos casos encontrados y desconocimiento del personal.

La escasa información en la literatura mundial acerca de este tipo de germen, las infecciones que produce, características epidemiológicas y mecanismos de resistencia, requiere sensibilización de los médicos en la presentación de infecciones emergentes, su reconocimiento y manejo adecuados. Cada vez nos enfrentamos a nuevos retos, es claro el perfil epidemiológico cambiante de las infecciones, gérmenes, perfiles de resistencia y factores relacionados.

\section{Agradecimientos}

Dra. María Isabel Múnera Jaramillo, médica microbióloga, coordinadora del área de microbiología, Hospital Pablo Tobón Uribe.

\section{Referencias}

1. Antunes MM, Antunes AA, Campos-Takaki GM. Incidence and resistance profile of Cedecea sp isolated from hospital. Laboratorio Central de Salud Pública - LACEN Recife/Brazil. Current Research Topics in Applied Microbiology and Microbial Biotechnology 2009; pp 510-514.

2. Grimont P, Grimont F, Farmer J and Asbury M. Cedecea davisae gen. nov., sp 
Nov. and Cedecea lapagei sp. Nov., New EnterobacteriaceaefronclinicalSpecimens. IJSEM 1981, 31 (3): 317-326.

3. Coudron PE, Markowitz SM. Cedecea lapagei isolated from lung tissue. ClinMicrobiolNews. 1987; 21 (9): 171-2.

4. Dalamaga M, Karmaniolas K, Arsenis G, et al. Cedecea lapagei bacteremia following cement-related chemical burn injury. Burns 2008; 34 (8): 1205-1207.
5. Hernandez M, Jiménez Arguedas G, Hernandez V, Herrera ML, Vargas A, Herrera JF. Ventriculitis por Cedecea lapagei: Reporte de un caso. Acta Pediatrcostarric. 2003; 17 (1): 29-31.

6. Ammenouche N, Dupont $\mathbf{H}$ and Mammeri. Characterization of a novel Amp C B-lactamase produced by carbapenem-resistant Cedecea davisae clinical isolate. HAAC 2014; 58 (11): 6942. 